\title{
Lytic Bone Lesions: Osteomyelitis or Intraosseous Gout?
}

JASON W. AN, MD, Department of Medicine, University of Alberta, Edmonton, Alberta, Canada; SARAH M. TROSTER, MD, FRCPC, Division of Rheumatology, University of Alberta, Edmonton, Alberta, Canada. Address correspondence to Dr. J. An, Department of Medicine, University of Alberta, 8440112 St. NW, Edmonton, Alberta T6G 2B7, Canada. E-mail: Jason.internalmed@gmail.com. Written informed consent was obtained from the patient. J Rheumatol 2017;44:1410-11; doi:10.3899/jrheum.170125

Dual-energy computed tomography (DECT) is a relatively new imaging technique that differentiates and color codes calcium (high attenuating) from urate (low attenuating) deposits in bone. Our report shows DECT distinguishing between tophaceous gout and other osteolytic lesions.

A 56-year-old woman presented with 2 weeks of acute chronic pain, redness, and swelling of the left foot, fifth metatarsal base at a site of a remote fracture. She had a presumed history of gout never confirmed with joint aspiration and a history of cardiorenal transplant, for which she was receiving immunosuppression therapy. Plain radiograph showed an aggressive mixed sclerotic and lucent lesion at the left fifth metatarsal base, appearing on gallium scan as nonspecific inflammation suspicious for osteomyelitis. DECT was performed, which revealed a large intraosseous tophaceous deposit within the base of the left fifth metatarsal (Figure 1). Aspiration produced a white crème-like fluid, negative gram stain and culture,

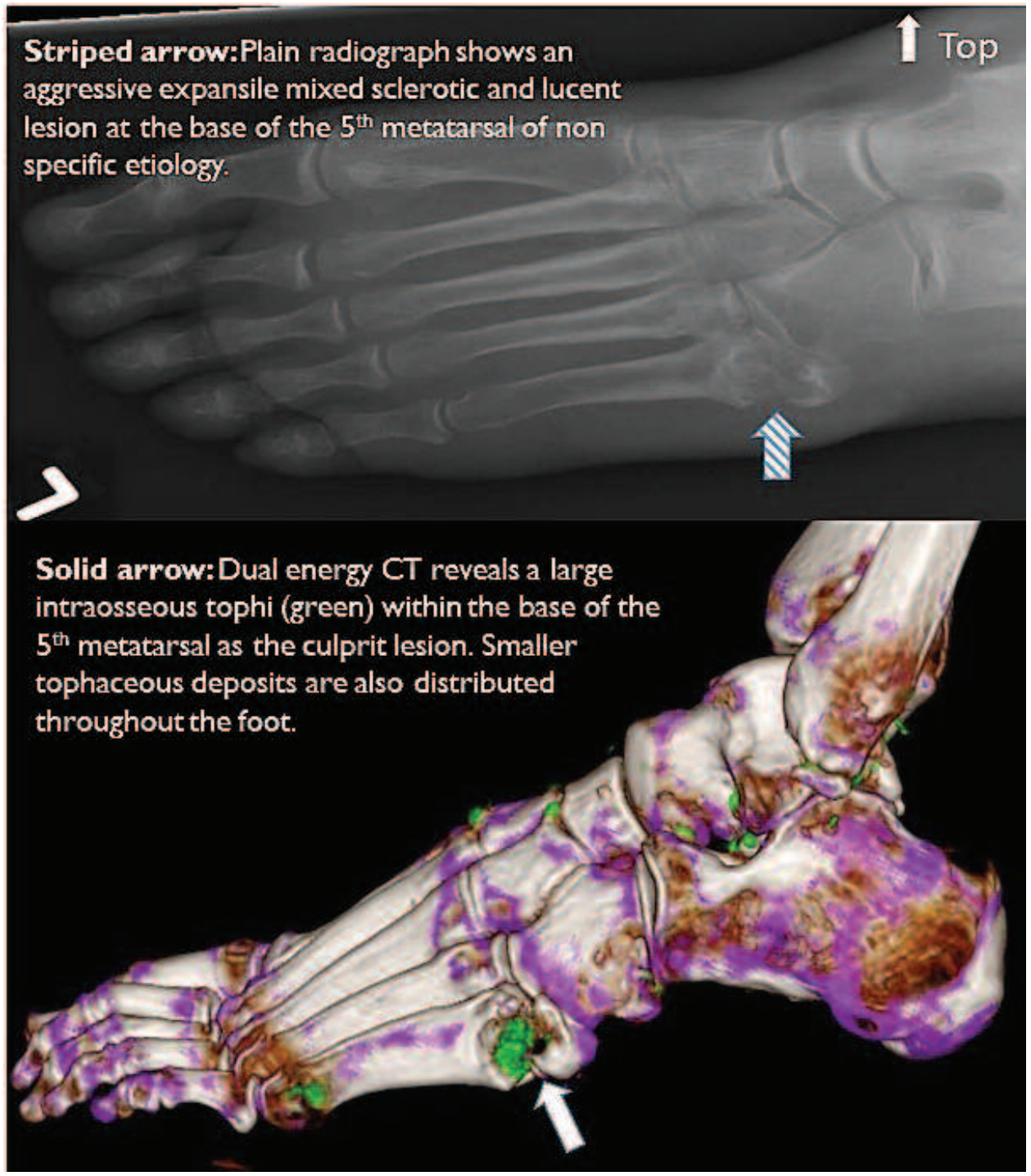

Figure 1. Radiograph shows lesion (top panel) and dual-energy CT shows tophi (bottom panel) in a left foot, fifth metatarsal base. CT: computed tomography. 
$4667 \times 10^{6} / 1$ white blood cells, and negatively birefringent crystals within acute inflammatory cells. The patient was diagnosed with gout complicated by intraosseous tophus invasion. Empiric antibiotics were discontinued and she was treated with prednisone and topical diclofenac with clinical improvement.

DECT has a sensitivity for urate crystals of up to $85 \%$ and a specificity of $93 \%{ }^{1}$. In a study, DECT identified $4 \times$ the quantity of tophaceous deposits compared with physical examination ${ }^{1}$, and in another was helpful in detecting intraosseous tophi, which cannot be seen on ultrasound ${ }^{2}$.
Our report highlights the involvement of a DECT scan in differentiating between tophaceous gout and other osteolytic lesions such as osteomyelitis in the appropriate clinical context.

\section{REFERENCES}

1. Hoi HK, Al-Arfaj AM, Eftekhari A, Munk PL, Shojania K, Reid G, et al. Dual energy computed tomography in tophaceous gout. Ann Rheum Dis 2009;68:1609-12.

2. Araujo EG, Manger B, Perez-Ruiz F, Thiele RG. Imaging of gout: new tools and biomarkers? Best Pract Res Clin Rheumatol 2016;30:638-52. 